\title{
TWO-DIMENSIONAL REPRESENTATIONS OF GROUPS WITH PROPERTY FA
}

\author{
ROGER C. ALPERIN
}

(Communicated by Warren J. Wong)

\begin{abstract}
We show that the two-dimensional representations of groups having property FA have finite image, if they always have compact closure.
\end{abstract}

In this note we generalize a theorem of Zimmer [Z; Theorem 7] that concerned groups having Kazhdan's property $T$ to the class of groups having property FA. It is known that a group having property $T$ also has property FA of Serre, $\left[\mathrm{W}_{1}\right]$. Where the results of $[\mathrm{Z}]$ relied on information concerning the decay of matrix entries of unitary representations of the given group, we shall instead use actions on trees.

Theorem. If $\Gamma$ is a finitely generated group of type FA such that every 2dimensional representation

$$
\rho: \Gamma \mapsto \mathrm{GL}_{2}(\mathbf{C})
$$

has image with compact closure, then $\rho(\Gamma)$ is finite for every two-dimensional representation.

The proof of the following corollary follows easily from the theorem.

Corollary. A finitely generated subgroup $\Gamma$ of $\mathrm{GL}_{2}(\mathbf{C})$ is finite if and only if (1) $\Gamma$ is of type FA and (2) every 2-dimensional representation

$$
\rho: \Gamma \mapsto \mathrm{GL}_{2}(\mathbf{C})
$$

has image with compact closure.

Proof of theorem. According to the reformulation and extension of Serre's FA property [B; Theorem 1] the subgroup $\rho(\Gamma)$ of $\mathrm{GL}_{2}(\mathbf{C})$ is either: (a) a free product with amalgamation; (b) has a nontrivial homomorphism to $\mathbf{Z}$; (c) conjugate to an upper triangular group with roots of unity on the diagonal; or (d) conjugate to a subgroup of $\mathrm{GL}_{2}(A)$ for a ring of integers $A$.

Received by the editors March 13, 1989 and, in revised form, April 3, 1989.

1980 Mathematics Subject Classification (1985 Revision). Primary 20E99, 20 H99.

Key words and phrases. Property FA .

Research partially supported by a grant from the National Security Agency. 
By property FA [S; Chapter 6], cases (a) and (b) cannot occur. In case (c) $\rho(\Gamma)$ has a subgroup of finite index which is unipotent; so $\rho(\Gamma)_{u}$ is trivial and $\rho(\Gamma)$ is finite. In case (d) since $\rho(\Gamma)$ has compact closure all of the eigenvalues have absolute value 1 . Furthermore, by use of the characteristic polynomial, we find that the eigenvalues of any element of $\rho(\Gamma)$ are in some integral quadratic extension $B$ of $A$; it follows from the compactness assumption on $\rho(\Gamma)$ that the eigenvalues must have absolute value one for every embedding of $B$ in $\mathbf{R}$ or C. Thus, it follows from the Dirichlet Unit Theorem that the eigenvalues of any given element are roots of unity. Moreover, for all elements of $\rho(\Gamma)$, these roots of unity have degree bounded by twice the dimension of the quotient number field of $A$ and hence the eigenvalues are finite in number. Now, Greenberg's Lemma [ $\mathrm{W}_{2}$; Lemma 4.5] asserts that a finitely generated linear group has a subgroup of finite index with traces (other than 2) not belonging to some given finite set; since there are finitely many roots of unity to avoid, and therefore finitely many traces to avoid, $\rho(\Gamma)$ has a subgroup of finite index whose eigenvalues are all 1 and therefore $\rho(\Gamma)$ is finite.

\section{REFERENCES}

[B] H. Bass, Finitely generated subgroups of $\mathrm{GL}_{2}$, The Smith Conjecture, edited by $\mathrm{H}$. Bass and J. W. Morgan, Academic Press, New York, 1984.

[S] J-P. Serre, Trees, Springer-Verlag, New York, 1980.

[ $\mathrm{W}_{1}$ ] Y. Watatani, Property $T$ of Kazhdan implies property FA of Serre, Math. Japonica 27 (1981), 97-103.

$\left[\mathrm{W}_{2}\right]$ B. A. F. Wehrfritz, Infinite linear groups, Springer-Verlag, New York, 1973.

[Z] R. Zimmer, Kazhdan groups acting on compact manifolds, Inventiones Math. 75 (1984), 425436.

Department of Mathematics and Computer Science, San Jose State University, SAN Jose, California 95192 